\title{
Einige Beziehungen zwischen den Zerfallsenergien der leichten radioaktiven Kerne
}

\author{
Von Hugo Neuert * \\ (Z. Naturforschg. 3a, 152-158 [1948]; eingegangen am 6. Dezember 1947)
}

\begin{abstract}
Es wird gezeigt, daß im Bereiche der leichten radioaktiven Kerne (bis etwa $A=40$ ) einige einfache Beziehungen zwischen Halbwertszeit, Zerfallsenergie und -art, Kernmasse und Kernspin bestehen. Teilt man die Bindungsenergien der radioaktiven Kerne in einen halbempirisch näherungsweise berechenbaren spinunabhängigen und einen spinabhängigen Energieanteil auf, so können aus den beobachteten Zerfallsenergien für einige Kernfamilien die spinabhängigen Anteile als Funktion der Kernmasse gefunden werden. Durch Inter- und Extrapolation erhält man aus diesen Beziehungen einige Hinweise hinsichtlich neuer oder bisher nur unvollständig bekannter Kerne im Bereiche bis $A=40$. In einem 2. Teil wird vorgeschlagen, die Differenzen zwischen den berechenbaren Bindungsenergien und den gemessenen Massendefekten bei den stabilen Kernen bzw. den beobachteten Zerfallsenergien bei den radioaktiven Kernen, als Energieanteile von bisher nicht erfaßten Kernkräften, in erster Linie wohl der Spinkräfte, anzusehen.
\end{abstract}

I.

$\mathrm{B}$ ei der großen Zahl der künstlich radioaktiven Kerne ist es naheliegend, nach Beziehungen zwischen den charakteristischen Daten derselben, nämlich Halbwertszeit, Zerfallsenergie und -art, Kernmasse und Kernspin, zu suchen. Die hier vorliegenden Betrachtungen sollen sich nur mit Beziehungen zwischen den leichten Kernen bis etwa $A=40$ befassen, da in diesem Bereich die experimentellen Daten einigermaßen vollständig sind. Zudem scheinen gerade hier einige einfache und zunächst nur in diesem Bereich gültige Beziehungen $\mathrm{zu}$ bestehen. Den folgenden Überlegungen liegen die Daten der kernphysikalischen Tabellen von Mattauch und Flügge ${ }^{1}$, die Tabellen in Ri e z le rs Einführung in die Kernphysik $^{2}$ sowie die Isotopentabellen von Se a bo rg ${ }^{3}$ zugrunde. Bei abweichenden Daten sind die Originalarbeiten zitiert ${ }^{4}$.

Es hat sich für die folgenden Betrachtungen als zweckmäßig erwiesen, die stabilen und die in-

* (17b) Weil a. Rh., Marktplatz 5.

1 J. M a tta u ch u. S. Flüg ge, Kernphysikalische Tabellen, Berlin 1942.

2 W. Riezler, Einführung in die Kernphysik, 3. Aufl., Leipzig 1944.

3 G. T. S e a b or g, Rev. mod. Physics 16, 1 [1944].

${ }^{4} \mathrm{H}^{3}$ nach R. J. Watt u. D. W. Williams, Physic. Rev. 70, 640 [1946]; $\mathrm{Li}^{8}$ nach W. H. B a r k a s, Physic. Rev. 72, 346 [1947]; Be ${ }^{10}$ nach E. M c M illan u. S. Ruben, Physic. Rev. 70, 123 [1946]; N16 nach S o m m e r u. S ch e r r e r, Physic. Rev. 69, 21 [1946]; $\mathrm{O}^{19}$ nach L. Sere n, W. L. Mayer u. W. St u r m, stabilen Kerne je nach ihrem Überschuß an Neutronen gegenüber der Zahl der Protonen zu klassifizieren. Es sei $N-Z=I$ die Zahl der Überschußneutronen. Unterscheidet man noch, ob eine gerade $(g)$ oder ungerade $(u)$ Zahl von Neutronen im Kern vorhanden ist, so erhält man bekanntlich Familien ( $I, g$ bzw. $I, u)$ von'Kernen mit ähnlichem Aufbau. Im allgemeinen werden die Kerne von $0, g ; 2, g ;-2, g$ usw. als ,doppelt gerade" Kerne, die Kerne von $-1, g ;-1, u ; 1, g$; $1, u ; 3, g$ usw. als ,ungerade" Kerne und die Kerne von $0, u ; 2, u ; 4, u$ usw. als ,doppelt ungerade" Kerne bezeichnet (vgl. Fleischmann ${ }^{4 a}$ ). Eine auffallende Eigenschaft der angeführten Kernfamilien ist die Ordnung hinsichtlich der Halbwertszeiten, d.h. der Stabilitäten der radioaktiven Kerne (vgl.z.B. auch $\left.{ }^{5}\right)$. Trägt man $\log _{10} t(t$ in sec) als Funktion der Kernmasse auf, so erhält man für die Werte der $\beta^{+}$-Strahler der Familien - $1, u$ und $-1, g$ eine Kurve, die sich recht gut durch eine Hyperbel etwa der Form $\left(\log _{10} t+1\right) \cdot A=48$

Physic. Rev. 69, 21 [1946]; Na ${ }^{24}$ nach C o r k, J u r $\mathrm{n}$ e y u. L an ge r, Physic. Rev. 70, 985 [1946]; $\mathrm{Al}^{28}$ nach E. Ble ule r u. W. Z ü nti, Helv. physica Acta 20, 195 [1947]; $\mathrm{Cl}^{34}$ nach Ho Z a h-W e i, Physic. Rev. 70, 782 [1946]; P34 nach E. B leuler u. W. $\mathrm{Z}$ ü n ti , Helv. physica Acta 19, 151 [1946]; K ${ }^{40}$ nach E. B l e u ler u. W. Gabri el, Helv. physica Acta 20, 67 [1947]; Cl ${ }^{38}$ nach K. S i e g bahn, Proc. Roy. Soc. [London] Ser. A, 188, 541 [1947]. [1940].

4a R. Fl e is chmann, Angew. Chem. 53, 486

${ }_{5}^{5}$ G. R. D i c ks on u. E. J. K on op inski, Physic. Rev. 58, 949 [1940]. 
darstellen läßt (Abb.1). Dagegen ist bei den $\beta+$ Strahlern von $0, u$ und $-2, g$ nicht ohne weiteres eine Gesetzmäßigkeit zu erkennen.

Sucht man eine ähnliche Beziehung für die $\beta^{-}$Strahler, so erhält man für die Werte der Familie $3, u$ mit ebenfalls guter Näherung eine der vorigen entgegengesetzt laufende Hyperbel (Abb.2). Auch

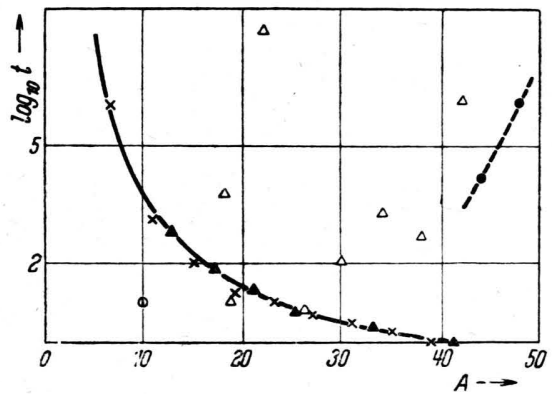

Abb. 1. Halbwertszeit der $\beta+$-Strahler als Funktion der Kernmasse.

Näherungskurve $\left(\log _{10} t+1\right) \cdot A=48$

$\times-1, g \Delta-1, g \quad \Delta 0, u \bigcirc 2, u \quad$ (1) $-2, g$

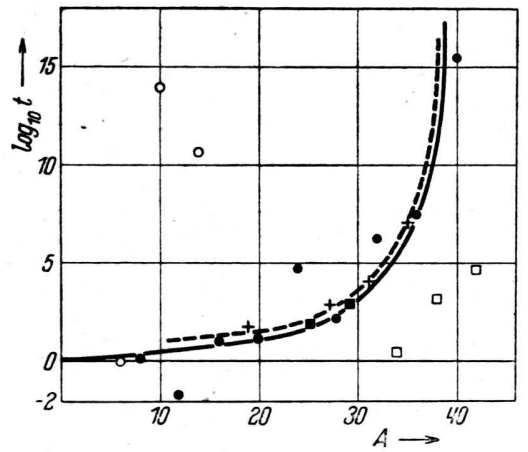

Abb. 2. Halbwertszeit der $\beta^{-}$-Strahler als Funktion

$$
\begin{gathered}
\multicolumn{2}{c}{\text { der Kernmasse. }} \\
\cline { 2 - 2 } \text { - Näherungskurven } \\
\hdashline 2, \boldsymbol{g}+3, u \quad \square, g \quad \square 4, g
\end{gathered}
$$

für 2, $u$ läßt sich eine solche Näherungskurve finden. Allerdings liegen hier einige Werte, nämlich für $\mathrm{B}^{12}, \mathrm{Na}^{24}, \mathrm{P}^{32}$ vollkommen außerhalb der Kurve. Die Werte von 3, $g$ liegen eher auf der Kurve für 2, $u$ als auf der für $3, g$.

Betrachtet man die in den Familien mit radioaktiven Kernen auftretenden Zerfallsenergien, so findet man eine ähnliche Ordnung. In den Abb. 3 und 4 sind die gemessenen Zerfallsenergien $E=E_{\text {kin }}(\beta)+E_{\gamma}$ in $\mathrm{MeV}$ als Funktion von $A$ aufgetragen. Im Falle der $\beta+$-Strahler von $-1, u$ und $-1, g$ findet man eine ziemlich glatte Kurve (Abb.3). Die Abweichungen der Meßwerte von der gezeichneten Näherungskurve sind gering. Die Übereinstimmung wäre noch vollkommener, wenn $\mathrm{P}^{29}, \mathrm{~S}^{31}$ und $\mathrm{Ar}^{35}$ eine um jeweils etwa

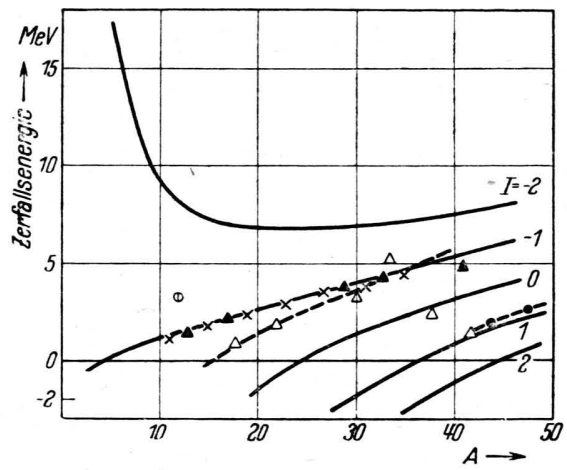

Abb. 3. Abhängigkeit der Zerfallsenergie von der Kernmasse für $\beta+$-Strahler.

Spin-unabhängige Energieanteile, Parameter $I$

(1) $-2, g \times-1, u \quad \Delta-1, g \quad \Delta 0, u \quad 2, u$

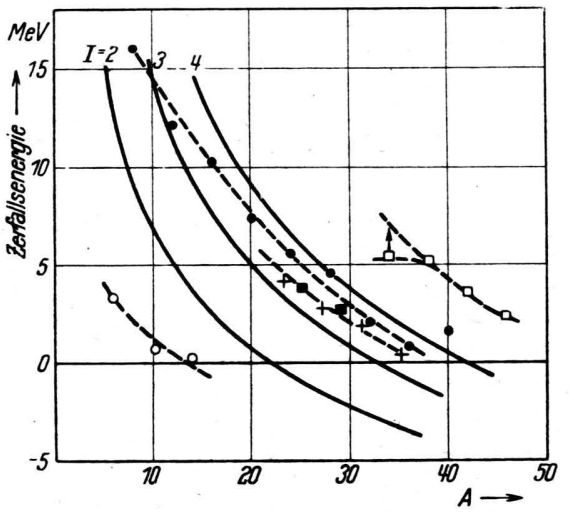

Abb. 4. Abhängigkeit der Zerfallsenergie von der Kernmasse für $\beta^{-}$-Strahler.

Spin-unabhängige Energieanteile, Parameter $I$

- $2, u \quad$ ○ $2, g+3, u \quad \square 3, g \quad \square 4, u$

0,2 $\mathrm{MeV}$ größere-Zerfallsenergie hätten (vgl. hierzu auch Jensen und Steinwed e ${ }^{6}$ ). Die Werte von $0, u$ (Abb.4) liegen zunächst auf einer glatten Kurve, zeigen aber später starke Abweichungen. Bei den $\beta^{-}$-Strahlern kann man ebenfalls für die verschiedenen Kerntypen gute Näherungskurven angeben.

Von den oben gezeigten Beziehungen ist diejenige zwischen der Zerfallsenergie und der

6 J. H. J e n s e n u. H. S t e in w e d e l, Naturwiss. 33, 249 [1946]. 
Massenzahl $A$ die aufschlußreichste. Einige der Werte sind, sowohl für die $\beta^{+-}$als auch für die $\beta^{-}$-Zerfälle, insbesondere hinsichtlich der Berechnung der Massendefekte der Kerne, schon mehrfach diskutiert worden ${ }^{7,7 a, 8-10}$. Man kann nun als Arbeitshypothese die Bindungsenergie eines Kernes in einen nur von $A, Z$ bzw. $I$ abhängigen Anteil $\varphi(Z, A)$ und einen nur vom Kernspin abhängigen Anteil $\chi(Z, A)$ trennen. Der spinunabhängige Anteil ist halbempirisch recht genau berechnet worden (vgl. z. B. ${ }^{1,7,7 a, 8}$ ). Utber den spinabhängigen Anteil ist nur wenig bekannt. Beim $\beta$-Zerfall muß nun die Differenz der Bindungsenergien des Ausgangs- und des Endkerns gleich der gesamten in Erscheinung tretenden Energie sein. Also

$$
E^{ \pm}=\varphi_{e}-\varphi_{a}+\chi_{e}-\chi_{a} .
$$

Für $\varphi_{e}-\varphi_{a}=\Delta \varphi$ ergibt sich aus der Formel für die Bindungsenergie für den $\beta^{-}$-Zerfall

$\Delta p^{-}=0,766+\frac{4 \beta(I-1)}{A}-\delta \frac{(A-I+1)}{A^{1 / 3}} \mathrm{MeV}$,

für den $\beta+$-Zerfall

$$
\begin{array}{rl}
\Delta \gamma^{+}=-0,766-2 m & m c^{2}-\frac{4 \beta(I+1)}{A} \\
& +\delta \frac{(A-I \quad 1)}{A^{1 / 3}} \mathrm{MeV} .
\end{array}
$$

Für $\beta$ nimmt man neuerdings den Wert 19,5 $\mathrm{MeV}^{10}$, für $\delta$ den Wert $0,6 \mathrm{MeV}$ an ${ }^{7,8}$. Für den Spin 0 kann $\operatorname{man} \chi=0$ setzen, also wahrscheinlich für alle Kerne mit geradem $I$ und geraden Neutronenzahlen, sicher aber für die Kerne $0, g$. Man kann daher bei solchen Zerfallsprozessen, bei denen der radioaktive oder der Endkern die Spinenergie 0 hat, aus der obigen Formel (1) direkt den spinabhängigen Anteil der Zerfallsenergie als Funktion der Kernmasse bestimmen. Die so erhaltenen Werte von $\chi$ seien im folgenden zusammengestellt unter der Annahme, daß auch die Kerne 2, $g$; $-2, g ; 4, g$ usw. den Spin 0 und $\chi=0$ besitzen:

1a. $2, u$ geht bis $\mathrm{K}^{40}$ durch $\beta^{-}$-Zerfall in $0, g$ uiber.

7 E. Wi g n e r, Physic. Rev. 51, 947 [1937].

7a C. F. v. We i zsäcker, Die Atomkerne, Leipzig 1937.

${ }_{5}$ H. B a rkas, Physic. Rev. 55, 691 [1939].

${ }^{9}$ H. A. Bethe u. R. F. B a cher, Rev. mod. Physics 9, 1 [1937].

${ }_{10}$ M. N. S a ha u. A. K. S a ha, Nature [London] 158, 6 [1946].
Also $E^{-}-\Delta \varphi^{-}(I=2)=-\chi_{2, u \rightarrow 0, g}$ bis $A=40$. 1b. $2, u$ geht ab $A=40$ durch $\beta+$-Zerfall in $4, g$ über.

Also $E^{+}-\Delta \varphi^{+}(I=2)=-\chi_{2, u \rightarrow 4, g}$ für $A \geqq 40$.

2. $0, u$ geht durch $\beta+$-Zerfall in $2, g$ über.

Also $E^{+}-\Delta \varphi^{+}(I=0)=-\chi_{0, u \rightarrow 2, g}$ ab $A=18$.

3. $4, u$ geht durch $\beta^{-}$-Zerfall in $2, g$ über.

Also $E^{-}-\Delta \varphi^{-}(I=4)=-\chi_{+, u \rightarrow 2, g}$ ab $A=34$.

4. 2,g geht durch $\beta^{-}$-Zerfall in $0, u$ über.

Also $E^{-}-\Delta \varphi^{-}(I=2)=\chi_{2, g \rightarrow 0, u}=-\chi_{0, u \rightarrow 2, g}$ bis $A=14$.

5. $-2, g$ geht durch $\beta^{+}$-Zerfall über in $0, u$.

$$
\begin{aligned}
& \text { Also } E^{+}-\Delta \varphi^{+}(I=-2)=\chi_{-2, g \rightarrow 0, u} \\
& =-\chi_{0, u \rightarrow-2, g} \text { für } A=10 \text {. }
\end{aligned}
$$

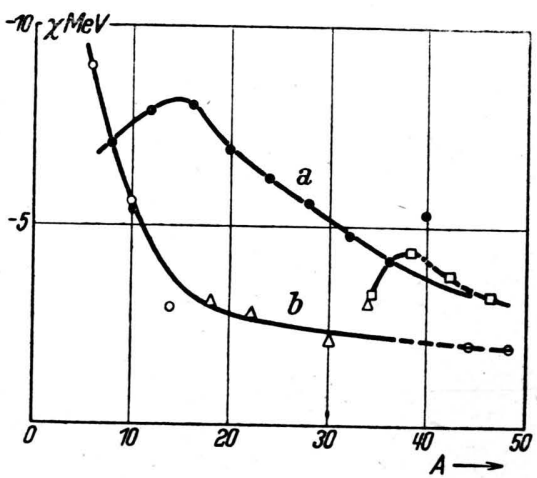

Abb. 5. Spinabhängige Anteile der Zerfallsenergie beim $\beta$-Zerfall.

$$
\begin{gathered}
2, u \rightarrow 0, g \quad \square 4, u \rightarrow 2, g \quad \triangle 0, u \rightarrow 2, g \\
\ominus 2, u \rightarrow 4, g \quad \bigcirc 2, g \rightarrow 0, u
\end{gathered}
$$

In den Abb. 3 und 4 sind neben den Näherungskurven für die experimentell bestimmten Werte $E \pm$ auch die berechneten Kurven für die $\Delta \varphi \pm(I, A)$ für verschiedene $I$ aufgezeichnet* Die aus der Differenz der gemessenen $E$-Kurven und den berechneten Kurven für $\Delta \varphi$ gefundenen Werte für $\chi$ sind in Abb. 5 aufgetragen. Sie sind alle negativ, wenn man die Werte für die Bindungsenergie positiv aufträgt. Die Punkte der Abb.5 gruppieren sich offenbar um 2 Näherungskurven a und b.

Die Kurve a wird in der Hauptsache aus den Werten $2, u$ gebildet, bei denen beim $\beta^{-}$-Zerfall

* Anm. b. d. Korrektur: Hr. Prof. F le i s c h m a n n hat mich freundlicherweise darauf aufmerksam gemacht, daß sich beide Figuren leicht $\mathrm{zu}$ einer einzigen vereinigen lassen. Es unterscheiden sich nämlich die Absolutwerte für $\Delta \varphi+$ für $I=k(k \mathrm{z} . \mathrm{B} .=0,1,2)$ und für $\Delta \varphi-$ für $I=k+2(k$ also z. B. $=2,3,4)$ nur um $2 m c^{2}$, so daß sich die Kurvenscharen der $\Delta \varphi^{+}$und $\Delta \varphi-$ z. B. durch Spiegeln der $\Delta \varphi-$ an der Abszisse und Verschiebung der Null-Linie um $2 m c^{2}$ zur Deckung bringen lassen. 
der vermutlich ganzzahlige Spin des Ausgangskerns ganz verschwindet. Bei den Endkernen $0, g$ sind die Spins der Einzelbausteine der Kerne gegenseitig vollständig abgesättigt; diese Kerne besitzen bekanntlich die größte Bindungsenergie. Die Spinenergie-Werte sind daher für die Kerne $2, u$ besonders groß. Für größere Massenzahlen $(A>35)$ geht die Kurve scheinbar in diejenige für den Zerfall der Kerne $4, u$ in $2, g$ über, bei dem durch den $\beta^{-}$-Zerfall der Spin wahrscheinlich ebenfalls verschwindet und der Neutronenüberschuß um den Betrag 2 abnimmt. $\mathrm{Al}^{24}$, von dem bisher nur die Zerfallszeit bekannt ist, ist wahrscheinlich auch in dieser Kurve enthalten. Die Kurve a gibt offenbar die Spinanteile für die Energieänderungen an, die auftreten bei den Um-

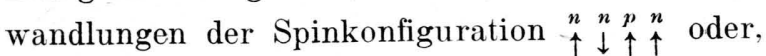
was hinsichtlich des Spinanteils dasselbe ist, der Anordnung $\begin{gathered}p p p n \\ \downarrow \uparrow \uparrow \uparrow\end{gathered} \quad$ in die Gruppierung $\begin{gathered}p n p n \\ \uparrow \uparrow \downarrow \downarrow\end{gathered}$.

Die Kurve a zeigt ein Maximum bei $A \sim 14$ und fällt nach kleinen Massen hin rasch, nach gröBeren Massen hin erst allmählich ab. Von der Kurve weichen die Werte für $\mathrm{P}^{34}$ und $\mathrm{K}^{40}$ erheblich ab. Bei $\mathrm{K}^{\mathbf{4 0}}$ hängt der höhere Spinenergieanteil wahrscheinlich mit dem hohen Spinübergang $(s=4)$ zusammen. Bei $\mathrm{P}^{34}$ ist das Zerfallsschema noch nicht vollständig geklärt ${ }^{1}$. Möglicherweise addiert sich die $\gamma$-Strahlung von $1,9 \mathrm{MeV}$ zu der beobachteten $\beta^{-}$-Strahlung von $5,1 \mathrm{MeV}$. Eine Zerfallsenergie von $6,5-7 \mathrm{MeV}$ würde sich gut in die Abb. 4 und 5 einfügen. Man kann allerdings auch vermuten, daß die $\chi$-Kurve für die Kerne 4, $u$ nach kleineren Massen hin umbiegt, wie es in der Abb. 5 gestrichelt angedeutet ist. Das würde erklären, daß unterhalb $\mathrm{P}^{34}$ keine Kerne dieser Familie bisher gefunden worden sind.

Die Kurve b enthält vor allem die Kerne 0, $u$, deren ganzzahliger Spin vermutlich verschwindet durch einen $\beta^{+}$-Zerfall in einen spinlosen Kern der Familie 2, $g$. Nach höheren $A$ hin schließen sich die Werte von $2, u$ an, die ebenfalls vermutlich durch $\beta^{+}$-Zerfall in einen Endkern $4, g$ ihren geradzahligen Spin abgeben. $\mathrm{C}^{10}$ kann offenbar mit $\mathrm{zu}$ der Kurve b gehörig betrachtet werden.

Bei den hier genannten Familien handelt es sich um die Spinanteile, die bei der Änderung der Spin-Konfiguration $\begin{gathered}p n \\ \uparrow \uparrow\end{gathered}$ in ${ }_{\downarrow \uparrow}^{n} n$ auftreten, und zwar in den Fällen, in denen diese dem spinlosen Kernrumpf $0, g$ ( $\alpha$-Teilchen-Kern) anhaften. Die $\mathrm{Zu}$ - stände $n, n$ sind aber nur bei den etwas schwereren Kernen $(A>16)$ stabil, da erst bei zunehmender Kernladung ein allmählich ansteigender Neutronenüberschuß zur Stabilität notwendig wird. Bei den leichtesten Kernen $(A<16)$ ist die $p{ }^{n}$-Konfiguration stabiler ( $\mathrm{Li}^{6}$ stabiler als $\mathrm{He}^{6}$ ), deswegen gehen bei kleinen Massenzahlen (bis $A=14$ ) nicht die Kerne $0, u$ in die Kerne 2, $g$, sondern vielmehr die Kerne 2, $g$ in die Kerne 0, $u$ über. Hinsichtlich des absoluten Betrages der Spinenergieanteile können diese Übergänge aber einfach als Fortsetzung der Übergänge von $0, u$ in $2, g$ betrachtet werden.

Zur Kurve b kann man wohl auch die Übergänge $2, u$ in $4, g$ (für $A \geqq 40$ ) rechnen, bei welchen bei gleichzeitigem Vorhandensein eines abgesättigten Neutronenpaares $\stackrel{n}{\uparrow} \downarrow^{n}$ ein Deuteron in eine zusätzliche Neutronengruppe übergeht. Bei 2, $u$ treten demnach für $A \leqq 40$ andere Umgruppierungen als für $A \geqq 40$. Man kann deshalb nicht einfach einer jeden Kerngruppe einen bestimmten Spinenergieanteil zuordnen. Es kommt vielmehr noch auf die Art des Übergangs an. Es sind deswegen bei den oben angeführten $\chi$-Werten noch die Anfangs- und Endfamilien durch Indizes angegeben.

Von der Kurve b weichen vor allem die Werte von $\mathrm{Cl}^{34}$ und $\mathrm{K}^{38}$ ab. Im Falle des $\mathrm{K}^{38}$ hat sich möglicherweise eine $\gamma$-Strahlung oder eine energiereiche $\beta$-Komponente bisher der Entdeckung entzogen. $\mathrm{Ob}$ die Kerne $\mathrm{Sc}^{44}$ und $\mathrm{V}^{48}$ wirklich mit auf der Kurve $b$ liegen, oder ob sie eher einer besonderen Kurve angehören, die bei $\mathrm{K}^{40} \mathrm{zu}$ etwas größeren Werten ansteigt, kann erst durch eine genaue Bestimmung der $\beta+$-Zerfallsenergie von $\mathrm{K}^{40}$ entschieden werden. Beim $\beta^{+}$-Zerfall der Kerne $-1, u$ und $-1, g$ mit halbzahligem Spin entstammt die Energie der $\beta^{+-S t r a h l e n ~ i m ~ w e s e n t-~}$ lichen allein dem Unterschied an Coulomb-Energie zwischen Ausgangs- und Endkern, eine Spinänderung wird wahrșcheinlich nicht eintreten ${ }^{11}$. Deswegen heben sich die spinabhängigen Anteile der Zerfallsenergien auf, d. h. es wird $E^{+}=\Delta \varphi^{+}$. Man sieht, daß die Werte für $-1, u$ und $-1, g$ praktisch ausnahmslos auf der Kurve für $\Delta \varphi^{+}$ $(I=-1)$ in Abb. 3 liegen.

Den obigen Kurven kann man nun eine Reihe von Hinweisen hinsichtlich neuer oder unvollstän-

11 M. G. Whit e, L. A. D e ls a s s o, J. G. Fox u. L. C. Creutz, Physic. Rev. 56, 512 [1939]; J. Schint $1 \mathrm{~m}$ e is ter, Z. Physik 122, 527 [1944]. 
dig bekannter radioaktiver Kerne im Bereiche bis etwa $A=40$ entnehmen. Einige solcher vermuteter Halbwertszeiten haben schon D i cks o n und Konopinski angegeben ${ }^{5}$. Auf einige auffallende Diskrepanzen mit den experimentellen Werten, z. B. bei $\mathrm{Ar}^{39}$, haben Saha und $\mathrm{Saha}{ }^{10}$ aufmerksam gemacht.

Vermutete neue Kerne:

\begin{tabular}{|c|c|c|c|c|}
\hline Isotop & $\begin{array}{l}\text { Kern- } \\
\text { typus }\end{array}$ & $\begin{array}{l}\text { Zerfalls- } \\
\text { art }\end{array}$ & $\begin{array}{c}\text { Halbwerts- } \\
\text { zeit }\end{array}$ & $\begin{array}{c}\text { Zerfalls- } \\
\text { energie } \\
\mathrm{MeV}\end{array}$ \\
\hline $\mathrm{C}^{15}$ & $3, \imath$ & ;- & $14 \mathrm{sec}$ & $9 \pm 1$ \\
\hline $\mathrm{N}^{17}$ & $3, g$ & ; & $8 \mathrm{sec}$ & $8 \pm 1$ \\
\hline $\mathrm{F}^{21}$ & $3, g$ & $\beta^{-}$ & $22 \mathrm{sec}$ & $5,5 \pm 0,5$ \\
\hline $\mathrm{Al}^{25}$ & $-1, g$ & + & $8 \pm 1 \mathrm{sec}$ & $3,5=0,5$ \\
\hline $\mathrm{Al}^{30}$ & $4, u$ & ; & $1 \mathrm{sec}$ & - \\
\hline $\mathrm{P}^{33}$ & $3, g$ & $a^{-}$ & $8,5 \mathrm{Stdn}$. & $1,1 \pm 0,5$ \\
\hline $\mathrm{K}^{37}$ & $-1, g$ & $b^{+}$ & $1 \mathrm{sec}$ & $4,9 \pm 0,5$ \\
\hline $\mathrm{Ar}^{3 \theta}$ & $3, u$ & $K$-Str. & groß & - \\
\hline
\end{tabular}

Bei $\mathrm{Al}^{26}(\beta+$ mit $t=7 \mathrm{sec})$. sind mehrere $\beta+$ Gruppen beobachtet worden. Die für $\mathrm{Al}^{25} \mathrm{zul}$ erwartende Halbwertszeit ist nun kaum von der für $\mathrm{Al}^{26}$ verschieden, so daß es wahrscheinlich ist, daß mindestens eine der dem $\mathrm{Al}^{26}$ zugeschriebenen Gruppen dem $\mathrm{Al}^{25}$ angehört. Der Wert für $\mathrm{Al}^{26}$ ist daher in den Abb. 3 und 5 nicht mit aufgezeichnet worden.

Unvollständig bekannte Kerne:

\begin{tabular}{|c|c|c|c|c|}
\hline Isotop & $\begin{array}{l}\text { Kern- } \\
\text { typus }\end{array}$ & $\begin{array}{c}\text { Zerfalls- } \\
\text { art }\end{array}$ & bekannt & $\begin{array}{c}\text { vermutet } \\
\mathrm{MeV}\end{array}$ \\
\hline $\mathrm{O}^{19}$ & $3, u$ & $\beta^{-}$ & $\left.\begin{array}{rl}t & =27 \mathrm{sec} \\
E_{\mathrm{kin}} & =3,3 \mathrm{MeV}\end{array}\right\}$ & $E=5,5 \pm 0,5$ \\
\hline $\mathrm{Na}^{21}$ & $-1, g$ & $r^{-}$ & $t=23 \mathrm{sec}$ & $E=2,6 \pm 0,3$ \\
\hline $\mathrm{Al}^{24}$ & $-2, u$ & $\beta^{+}$ & $t=20 \mathrm{~min}$ & $E=5,5 \pm 0,5$ \\
\hline $\mathrm{Cl}^{34}$ & $0, u$ & $p^{+}$ & $\left.\begin{array}{rl}t & =32 \min \\
E_{\mathrm{kin}} & =5,1 \mathrm{MeV}\end{array}\right\}$ & $E=4,2 \pm 0,5$ \\
\hline $\mathrm{K}^{38}$ & $0, u$ & $p^{+}$ & $\left.\begin{array}{rl}t & =7,65 \mathrm{sec} \\
E_{\mathrm{kin}} & =2,3 \mathrm{MeV}\end{array}\right\}$ & $E=5,0 \pm 0,5$ \\
\hline $\mathrm{Ca}^{39}$ & $-1, u$ & $p^{+}$ & $t=1,06 \mathrm{sec}$ & $E=5,1 \pm 0,5$ \\
\hline
\end{tabular}

II.

Im I. Abschnitt war die Bindungsenergie eines Kerns dargestellt worden durch

$$
\Delta M=\varphi(A, Z)+\chi(A, Z) .
$$

Dabei sollte $\varphi(A, Z)$ die vom Kernspin unabhängige Bindungsenergie, $\chi(A, Z)$ die vom Spin abhängige Energie ausdrücken. $\varphi$ kann z. B. (vgl. ${ }^{1,10}$ ) näherungsweise nach folgender Formel berechnet werden:

$$
\begin{aligned}
\varphi(A, Z)=+14,66 A & -19,5 \frac{(N-Z)^{2}}{A} \\
& -15,4 A^{2 / 3}-0,6 \frac{Z^{2}}{A^{1 / 3}} ;
\end{aligned}
$$

dabei ist $\varphi$, wie auch oben, positiv gerechnet worden. In Formel (4) sind die spinabhängigen Teile der Bindungsenergie noch nicht berücksichtigt worden. Es wird nun vorgeschlagen, die Unterschiede zwischen den nach (4) berechneten Bindungsenergien und den experimentell bestimmten Massenzahlen gleich den oben eingeführten Energieanteilen $\chi$ zu setzen. Also

$$
\Delta M_{\mathrm{gem}}-\varphi(A, Z)=\%(A, Z) .
$$

Hierbei sollen nun unter $\chi(A, Z)$ alle Energieanteile verstanden werden, die in (4) nicht enthalten sind, also in der Hauptsache die spinabhängigen Anteile, aber möglicherweise auch noch andere, bisher noch nicht quantitativ erfaßte Anteile. Die Anwendbarkeit der Formel (5) wäre gerechtfertigt, wenn in den Fällen, in denen der Spin mit Sicherheit gleich Null ist, $\chi$ ebenfalls gleich Null oder wenigstens sehr klein werden würde (z. B. im Falle $\mathrm{O}^{16}$ oder $\mathrm{Mg}^{24}$ ). Vergleicht man jedoch die gemessenen Massendefekte mit den aus (4) berechneten Bindungsenergien für die Kerne 0,g, so stellt man fest, daß gerade bei diesen Kernen die Abweichungen der beiden Werte voneinander besonders groß sind. Es scheint also nicht möglich zu sein, die Formel (5) zu einer einfachen Berechnung von $\chi$ heranzuziehen, wenigstens nicht im Bereiche der hier zunächst interessierenden leichten Kerne. In einigen anderen Fällen, $z_{\triangleleft} B$. bei den Kernen $1, g$ und $1, u$, stimmen die experimentellen und die nach (4) berechneten Energien recht gut überein. Das sind aber gerade die Kerne, bei denen man eine beträchtliche Differenz zwischen (4) und $\Delta M_{\text {gem }}$ erwarten sollte. Man kann daher wohl annehmen, daß mit Hilfe der Formel (4) zwar die Änderung der Bindungsenergie als Funktion von $Z, A$ recht gut bestimmt werden kann, daß der absolute Betrag aber zumindest im Gebiet der leichten Kerne nicht ganz richtig wiedergegeben wird.

Diese Schwierigkeit kann man dadurch umgehen, daß man nur nach der Änderung der Bindungsenergie fragt bei Änderung der Kernmasse um den Betrag 1, also bei der Anlagerung eines Neutrons oder eines Protons. Da dann nur die Differenzen der berechenbaren Bindungsenergien 
auftreten, heben sich die Unsicherheiten in der absoluten Höhe derselben mit einer gewissen Näherung heraus: Das hiermit vorgeschlagene Verfahren zur Ermittlung der Anteile $\chi$ der Kernbindungsenergie kann zwar damit nicht den Anspruch auf gute Genauigkeit in der Bestimming dieser Energie erheben. Man wird aber immerhin den wesentlichen Verlauf derselben für die einzelnen Kernfamilien in Abhängigkeit von $A$ auffinden können.

Die Anteile $\chi$ werden im einzelnen nach folgendem Schema berechnet:

$$
\begin{array}{r}
M(A, Z) \pm n+\varphi(A, Z)+\chi(A, Z)=M(A \pm 1, Z) \\
+\varphi(A \pm 1, Z)+\psi(A \pm 1, Z) \\
\chi(A \pm 1, Z)-\psi(A, Z)=M(A, Z) \pm n-M(A \pm 1, Z) \\
+\varphi(A, Z)-\varphi(A \pm 1, Z)
\end{array}
$$

und

$$
\begin{gathered}
M(A, Z) \pm p+\varphi(A, Z)+\chi(A, Z)=M(A \pm 1, Z \pm 1) \\
+\varphi(A \pm 1, Z \pm 1)+\chi(A \pm 1, Z \pm 1), \\
\chi(A \pm 1, Z \pm 1)-\psi(A, Z)=M(A, Z) \pm p \\
-M(A \pm 1, Z \pm 1)+\varphi(A, Z)-\varphi(A \pm 1, Z \pm 1) .(7)
\end{gathered}
$$

Die Differenzen der Massen sind hierbei aus den massenspektroskopischen Daten sehr genau und aus den Zerfallsenergien der radioaktiven Kerne mit hinreichender Genauigkeit bekannt. Die genauesten Werte für $\chi$ erhält man natürlich dann, wenn der eine der beiden $\chi$-Werte in (6) oder (7) gleich Null gesetzt werden kann, also mit Sicherheit im Falle der Kerne 0,g. Einige Kernfamilien können nur mit Hilfe der zuvor berechneten $\chi$-Werte anderer Familien berechnet werden. Die so erhaltenen $\chi$ sind daher mitunter weniger genau. Insbesondere sind die Werte um $\mathrm{O}^{18}$ mit einer Ungenauigkeit von etwa $1 \mathrm{MeV}$ behaftet. Diese scheint aber wohl auf eine bisher noch nicht erklärte Unregelmäßigkeit der der Rechnung zugrunde liegenden Kurven für die Atomgewichte im Bereiche der Kernmassen 16-20 zurückżuführen zu sein. Dieselbe tritt z. B. auch deutlich in den Abb. 11 u. 12 der oben schon erwähnten Arbeit von Fleischmann ${ }^{4 a}$ in Erscheinung.

Auf diese Weise wurden zunächst die $\chi$-Energien für die Kerne von $1, u$ und $-1, g$ sowie von $-1, u$ und $1, g$ berechnet. Alle Werte sind negativ. Sie sind in Abb. $6 \mathrm{a}$ und $\mathrm{b}$ aufgetragen für die leichten Kerne $(A=35)$. Für die schwereren Kerne sind die Kernmassen noch nicht genügend genau und vollständig bekannt. Die Kurven zei- gen, daß die zusätzlichen $\chi$-Energien bei den leichtesten Kernen am größten sind und dann auf einen Mittelwert von $2-3 \mathrm{MeV}$ bei $A \sim 35$ zurückgehen. Bemerkenswert ist die gezackte Form der Kurven. Ferner fällt die gute Übereinstimmung der Kurven für $-1, u$ und $1, g$ bzw. $1, u$ und

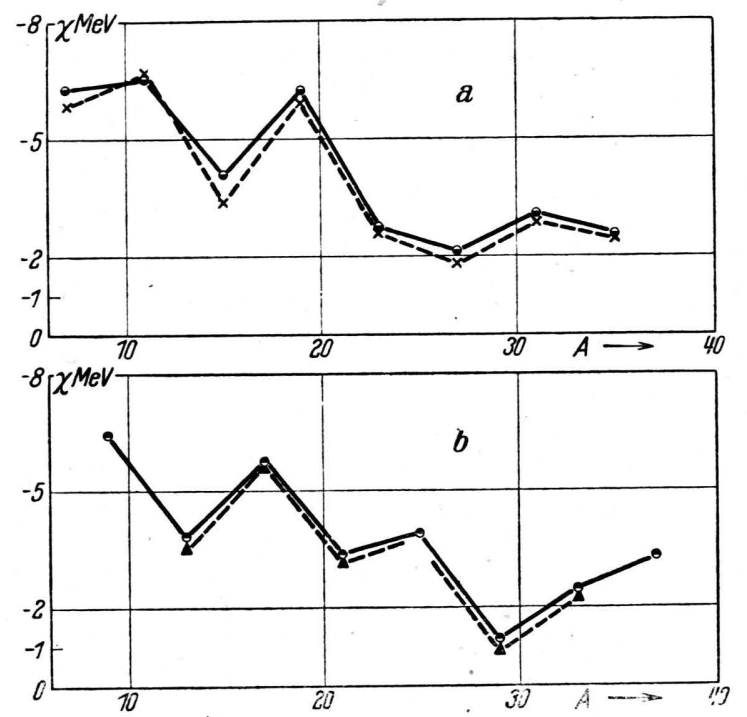

Abb. 6. Spinabhängige Anteile der Kernbindungsenergie.
a) $\times-1, u$
- $1, g$
b) $\odot 1, u$
$\Delta 1, g$

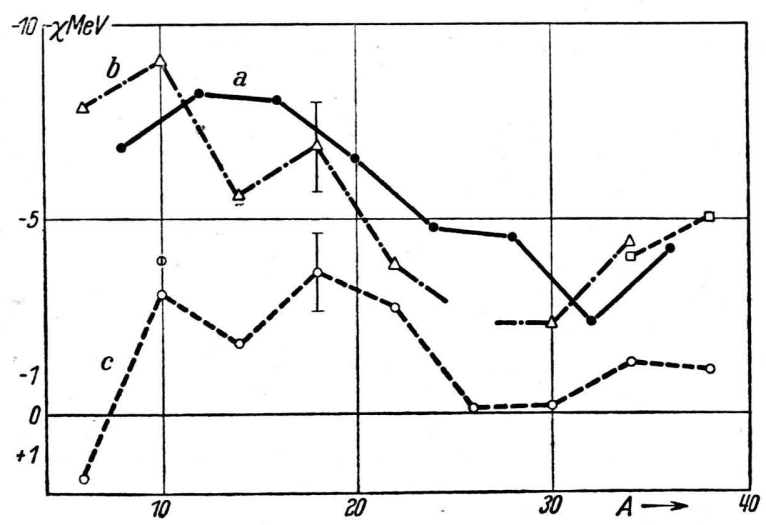

Abb. 7. Spinabhängige Anteile der Kernbindungsenergie.
$2, g$
(1) $-2, g$
$\triangle 0, u$
$2, u \quad \square 4, u$

$-1, g$ auf. Die Familien $-1, u$ und $1, g$ sowie $1, u$ und $-1, g$ haben offenbar einen durchaus ähnlichen Aufbau. Sie besitzen daher auch vermutlich entsprechende halbzahlige Kerndrehimpuls-Quantenzahlen.

Die $\chi$-Werte der Familie $2, u$ kann man mit Hilfe der schon bekannten Werte von $1, g$ berechnen (Abb. 7, Kurve a). Die $\chi$-Kurve für 2, $u$ ist im vorigen Abschnitt bereits auf andere Weise ermit- 
- telt worden (Kurve a in Abb. 5). Die Úbereinstimmung der beiden Kurven ist recht gut. Für die $\chi^{-}$ Werte der Familie 0, $u$ würde man in entsprechender Weise die Kurve b der Abb. 5 erwarten. Man erhält aber, wie Kurve b in Abb. 7 zeigt, im allgemeinen wesentlich höhere Werte. Bei der Berechnung der Kurve b in Abb. 5 war man davon ausgegangen, daß die Kerne von $0, u$ in solche Kerne zerfallen $(2, g)$, die vermutlich den Spin Null haben und denen deswegen auch der Wert $\chi=0$ zugeschrieben wurde. Wenn man nun aber nach dem hier genannten Verfahren $\chi$-Werte für $2, g$ ausrechnet, so erhält man die Werte der Kurve c in Abb. 7. Hiernach haben die Kerne $\mathrm{Be}^{10}$, $\mathrm{C}^{14}$, $\mathrm{O}^{18}$ und $\mathrm{Ne}^{22} \chi$-Energien von $2-3 \mathrm{MeV}$. Erst ab $\mathrm{Mg}^{26}$ ist auch $\chi=0$ oder nur wenig von Null verschieden. Die Kurve b in Abb. 5 gibt dann recht gut die Differenzen zwischen den Kurven b und c der Abb. 7 wieder. Die Spinenergie von $\mathrm{C}^{10}$ liegt wohl innerhalb der Fehlergrenze mit auf der Kurve c.

Dieses Resultat ist überraschend, da man bisher annehmen mußte, daß die Kerne 2, $g$ mit $s=0$ auch wirklich die Spinenergie 0 besitzen. Es erhebt sich daher erneut die Frage, ob den Kernen $\mathrm{Be}^{10}, \mathrm{C}^{14}, \mathrm{O}^{18}$ und $\mathrm{Ne}^{22}$ wirklich sowohl die Eigendrehimpuls-Quantenzahl 0 als auch die Bahndrehimpuls-Quantenzahl 0 zuzuordnen ist. Aus der weitgehenden Ahnlichkeit der Kurven für 1, $u$ und $-1, g$ in Abb. 6 b kann man ohne großen Fehler eine $\chi$-Energie für $\mathrm{Al}^{25}$ von $3,6 \pm 0,2 \mathrm{MeV}$ ableiten. Mit diesem Werte ergibt sich aus $\mathrm{Mg}^{24}+p$ ein Wert für die Masse des oben schon vermuteten $\mathrm{Al}^{25}$ mit etwa 8 sec Halbwertszeit von 24,9991 . Die maximal mögliche kinetische Energie der $\beta$ Strahlen wäre dann etwa $3,2 \pm 0,2 \mathrm{MeV}$, durchaus in guter Übereinstimmung mit dem aus Abb. 3 interpolierten Werte von $3,5 \pm 0,3 \mathrm{MeV}$. Für $\mathrm{Al}^{26}$ kann man aus Kurve b der Abb. 7 mit etwas geringerer Genauigkeit einen $\chi$-Wert von $2,3 \pm 0,5 \mathrm{MeV}$ ablesen. Mit Hilfe von $\mathrm{Al}^{26}+n=\mathrm{Al}^{27}$ und unter Benutzung des Wertes von $2,0 \mathrm{MeV}$ für $\mathrm{Al}^{27}$ erhält man für die Masse von $\mathrm{Al}^{26}$ den Wert 25,9933 $\pm 0,5 \mathrm{TME}$. Die maximale $\beta+$-Energie könnte dann $1,9 \pm 0,5 \mathrm{MeV}$ betragen.

\title{
Über die statistische Mechanik regulärer und irregulärer Lösungen
}

\author{
Von Arnold MÜnster \\ Aus dem Chemischen Institut der Universität Heidelberg \\ (Z. Naturforschg. 3 a, 158-172 [1948]; eingegangen am 10. Januar 1948)
}

\begin{abstract}
Es wird eine Methode entwickelt, die unter gewissen Voraussetzungen eine näherungsweise Berechnung der Verteilungsfunktion flüssiger Gemische von beliebig vielen Komponenten beliebiger Molekülgröße und -gestalt ermöglicht. Vorausgesetzt wird, daß 1. eine Komponente, das Lösungsmittel, zu mehr als 50\% der Gesamtmasse vorhanden ist, 2. die Moleküle des Lösungsmittels nicht größer als die der übrigen Komponenten und annähernd kugelförmig sind, 3. zwischen den Molekülen keine weitreichenden Kräfte auftreten. Das Ergebnis wird auf die Probleme der streng regulären binären Lösung sowie der mono- und polydispersen Lösung hochpolymerer Fadenmoleküle angewandt. Im letzteren Falle ergibt sich, daß die thermodynamischen Eigenschaften im allgemeinen von der Verteilung der Polymerisationsgrade abhängen.
\end{abstract}

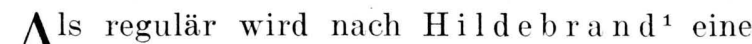
Alösung bezeichnet, deren Verdünnungswärme

$$
\Delta \mathrm{H}_{2} \neq 0
$$

ist, während für die Verdünnungsentropie

$$
\Delta s_{2}=-R \ln v_{2},
$$

$\left(s_{2}=\right.$ Molenbruch des Lösungsmittels LM, $R=$ Gaskonstante) gilt. Dagegen gilt bei irregulären

1 J. S. H i l d e brand, J. Amer. chem. Soc. 51, 66 [1929].
Lösungen die Beziehung (1); es ist aber

$$
\Delta s_{2} \neq-R \ln x_{2} \text {. }
$$

Das Auftreten einer von Null verschiedenen Verdünnungswärme beruht auf zwischenmolekularen Wechselwirkungen, die man gewöhnlich unter dem Begriff der Solvatation zusammenfaßt. In der statistischen Mechanik ist dieselbe dadurch definiert, daß für eine beliebige Konfiguration des Systems die Vertauschung zweier verschiedener Moleküle eine Änderung der potentiel- 